\title{
A PERCEPTUALLY ADAPTIVE APPROACH TO IMAGE DENOISING USING ANISOTROPIC NON-LOCAL MEANS
}

\author{
Alexander Wong, Paul Fieguth, and David Clausi \\ University of Waterloo \\ Department of Systems Design Engineering \\ Waterloo, Ontario, Canada
}

\begin{abstract}
This paper introduces a novel perceptually adaptive approach to image denoising using anisotropic non-local means. In the classical non-local means image denoising approach, the value of a pixel is determined based on the weighted average of other pixels, where the weights are determined based on a fixed isotropically weighted similarity function between the local neighborhoods. In the proposed algorithm, we demonstrate that noticeably improved perceptual quality can be achieved through the use of adaptive anisotropically weighted similarity functions between local neighborhoods. This is accomplished by adapting the similarity weighing function in an anisotropic manner based on the perceptual characteristics of the underlying image content derived efficiently based on the Mexican Hat wavelet. Experimental results show that the proposed method can be used to provide improved perceptual quality in the denoised image both quantitatively and qualitatively when compared to existing methods.
\end{abstract}

Index Terms - image denoising, anisotropic, non-local

One of the most fundamental areas of research in the field of image processing is image denoising, where an estimate of the original image is obtained by suppressing noise caused by various factors during the image acquisition process. Good image noise suppression is important for both aesthetic reasons and a functional reasons (e.g., computer vision applications such as object recognition and tracking). Given the fact that image denoising has been one of the fundamental tasks in image processing, many image denoising techniques have been proposed over the years. These can generally be categorized as either a spatial domain denoising method or transform domain denoising method. The first image denoising methods have been spatial domain denoising methods, which alter the intensity of a pixel directly in the spatial domain. Traditional techniques such as box filtering and Gaussian filtering treat all pixels equally during the denoising process, which leads to the unwanted blurring of perceptually significant characteristics. Newer spatial domain denoising techniques attempt to suppress noise while preserving such image characteristics. These newer denoising techniques include total variation [2], anisotropic filtering [1], bilateral filtering $[3,4]$, and non-local means filtering techniques $[5,6]$. Transform domain denoising techniques are those that first convert an image into a transform domain and then alters the transform coefficients to reduce noise. Some transform domain techniques include Wiener filtering [7] and waveletbased techniques $[8,9]$.

A state-of-the-art image denoising technique that has been introduced recently is the non-local means filtering method proposed by Buades et al. [5], which has been shown to produce state-of-the-art results compared to existing techniques. What is particularly interesting about the non-local means filtering method is the fact that, unlike existing techniques that rely on local statistics within a small neighborhood of pixels to suppress noise, it utilizes global statistics from the entire image. As such, the non-local means filtering method relies on the global self-redundancy of an image to reduce noise. This is particularly effective for the purpose of texture denoising since textures are typically highly redundant. While attempts have been made to improve the computational performance of the non-local means denoising algorithm [6], little effort has been placed on improving the visual quality of denoised images from a perceptual perspective. One of the biggest issues with the non-local means filtering method is that it utilizes a fixed isotropic weighing scheme when determining the denoised value of a pixel. As such, perceptually insignificant image content are often given too much weight in determining the value of perceptually significant image content. This leads to undesired image artifacts that degrade the perceptual quality of an image. The goal of this paper is to alleviate this problem through the use of adaptive anisotropic non-local means based on the perceptual characteristics of the underlying image content.

This paper is organized as follows. An overview of the non-local means image denoising method is described in 1 . The proposed method of using adaptive anisotropically weighted non-local means is described in 2. Experimental results are presented and discussed in Section 3. Finally, conclusions are drawn in Section 4. 


\section{IMAGE DENOISING USING NON-LOCAL MEANS}

Prior to describing the proposed method, it is important to first provide an overview of the non-local means image denoising method proposed by Buades et al. [5]. The non-local means method is unique from existing denoising methods in that it utilizes both local and global spatial statistics and global amplitudinal statistics in computing the value of a restored pixel within an image. Consider that an image $I_{o}$ is corrupted by noise to form a noisy image $I_{n}$. This can be formulated as follows:

$$
I_{n}(\underline{\mathrm{x}})=n\left(I_{o}(\underline{\mathrm{x}})\right)
$$

where $\underline{\mathrm{x}}=(x, y)$ and $n$ is the noise function. In the non-local means image denoising method, an estimate of the original image $I_{o}$ is defined as a weighted average of all pixels within the noisy image $I_{n}$. This can be expressed for a particular point $\underline{l}$ as follows:

$$
\hat{f}(\underline{x})=\sum_{\underline{m} \in I_{n}} w(\underline{l}, \underline{m}) I_{n}(\underline{m})
$$

where $w(\underline{l}, \underline{m})$ is an isotropic weighing function that evaluates the similarity between to the local neighborhoods of two pixels at $\underline{l}$ and $\underline{m}$ respectively. The isotropic weighing function be expressed as follows:

$$
w(\underline{l}, \underline{m})=\frac{1}{Z(\underline{l})} e^{-\frac{\left(\frac{1}{2 \pi \sigma} e^{-\left(\frac{(\varsigma)}{2 \sigma^{2}}\right)}\left\|I_{n}\left(\varsigma_{l}\right)-I_{n}\left(\varsigma_{m}\right)\right\|_{2}^{2}\right)}{h^{2}}}
$$

where $\varsigma_{l}$ and $\varsigma_{m}$ represents the local neighborhood around $\underline{l}$ and $\underline{m}$ respectively, $\varsigma$ represents a region with the same neighborhood size as $\varsigma_{l}$ and $\varsigma_{m}, h$ is the decay coefficient, and $Z(\underline{l})$ is a normalization term and is defined as $Z(\underline{l})=\sum_{\underline{m}} w(\underline{l}, \underline{m})$. The main advantage of the non-local means denoising method is that, unlike existing denoising techniques, it is not limited to local statistics around a particular pixel. Instead, this method is able to leverage all of the information within the image to compute an estimate of the pixel in its original form. This technique is particularly powerful in situations where the noisy images are characterized by high self-similarity from a global perspective. For example, an image texture may contain a high number of repeated patterns from a global perspective, but may be very unique from a local perspective. Existing techniques would have difficulty suppressing noise in this situation since it can only rely on local statistics. However, the non-local means denoising method is able to leverage the information from the repeated patterns throughout the image to better suppress image noise.

The main issue with non-local means denoising is that it utilizes an isotropic weighing function that is constrained by a fixed set of parameters. This can lead to undesirable visual degradation and artifacts for several reasons. First, the use of a fixed set of parameters for defining the weighing function means that the similarity between all local neighborhoods are evaluated the same way. In situations characterized by high levels of noise, the similarity between regions that are highly similar in the original image $I_{o}$ may become significantly lower in the noisy image $I_{n}$. One approach to achieving improved noise suppression in such situations is to increase the spatial spread $\sigma$ such that the weights between these similar regions is increased. While increasing the spatial spread is suitable for regions where there is little perceptually significant characteristics, this can lead to noticeable perceptual degradation in regions with perceptually significant characteristics due to oversmoothing. Therefore, a method for controlling the spatial spread based on the perceptual characteristics of the underlying image content is desirable.

A more important issue with the weighing function used in the non-local means image denoising method is the fact that it is isotropic in nature. What this means is that it does not account for the underlying perceptual characteristics of the image as it treats all pixels that are equidistant from the center of a neighborhood in the same manner. While this is reasonable for regions with few perceptually significant characteristics, it can lead to significant degradation in perceptual quality by overweighing the contribution of perceptually insignificant image content in determining the value of perceptually significant image content. One effective approach to addressing this issue is to use an anisotropic weighing function that is oriented and fitted along perceptually significant characteristics such as edges. This reduces the amount of insignificant perceptual characteristics that contributes to determining the similarity between local neighborhoods, thus reducing the impact of insignificant perceptual characteristics on the weighing function for regions containing significant perceptual characteristics. The proposed denoising algorithm utilizes the concept of perceptually adaptive anisotropic nonlocal means to address the aforementioned issues in hopes of suppressing image noise while preserving perceptually significant image characteristics.

\section{PERCEPTUALLY ADAPTIVE ANISOTROPIC NON-LOCAL MEANS}

The primary goal of the proposed denoising algorithm is to adapt the non-local means weighing function in an anisotropic manner based on the perceptual characteristics of the underlying image content. As such, it is necessary to define an objective function for measuring the perceptual significance of image content. In the proposed algorithm, an objective measure of the perceptual significance at a particular point $\underline{x}=(x, y)$ is defined based on the Mexican hat wavelet function: 


$$
\kappa(\underline{x})=\frac{\sqrt{\left(\varphi_{x}(\underline{x}) * I_{n}(\underline{x})\right)^{2}+\left(\varphi_{y}(\underline{x}) * I_{n}(\underline{x})\right)^{2}}}{\kappa_{\max }}
$$

where $\kappa_{\max }$ is the maximum value of $\kappa$ for the image and

$$
\varphi_{x}(x)=\frac{1}{\sqrt{2 \pi} \sigma_{s}^{3}} x e^{\frac{-x^{2}}{2 \sigma_{s}^{2}}}
$$

and

$$
\varphi_{y}=\varphi_{x}^{\mathrm{T}}
$$

Based on this objective measure of perceptual significance, the spatial spread of the weighing function can be adapted to achieve better perceptual quality based on the underlying image content. Image noise is highly noticeable in regions with few perceptually significant characteristics, such as smooth regions. As such, the spatial spread $\sigma$ described in (3) should be high enough to suppress image noise in these regions using the contributions of other pixels in the image. On the other hand, image noise is less noticeable in regions with a lot of perceptually significant characteristics. However, it is necessary to preserve structural detail in such regions as the human vision system is highly sensitive to degradation in such regions. Therefore, the spatial spread $\sigma$ should be low enough such that only pixels with high similarity has noticeable contribution to determining the restored values of pixels in these regions.

To adapt the weighing function in an anisotropic manner, it is necessary to determine the orientations of the underlying image content. In the proposed algorithm, the orientation at a particular point $\underline{x}$ is defined as follows:

$$
\theta(\underline{x})=\arctan \left[\frac{\left(\varphi_{y}(\underline{x}) * I_{n}(\underline{x})\right)}{\left(\varphi_{x}(\underline{x}) * I_{n}(\underline{x})\right)}\right]
$$

Based on the orientation as well as the aforementioned measure of perceptual significance, the shape and orientation of the weighing function can be adapted to provide a better fit the structural characteristics of the underlying image content.

Based on these observations, the proposed anisotropic weighing function for the non-local means denoising algorithm can be defined as follows:

$$
w(\underline{l}, \underline{m})=\frac{1}{Z(\underline{l})} e^{-\frac{\left(\psi(\kappa, \theta)\left\|I_{n}\left(\varsigma_{l}\right)-I_{n}\left(\varsigma_{m}\right)\right\|_{2}^{2}\right)}{h^{2}}}
$$

where $\varsigma_{l}$ and $\varsigma_{m}$ represents the local neighborhood around $\underline{l}$ and $\underline{m}$ respectively, $\varsigma$ represents a region with the same neighborhood size as $\varsigma_{l}$ and $\varsigma_{m}$, and $Z(\underline{l})$ is a normalization term and is defined as $Z(\underline{l})=\sum_{\underline{m}} w(\underline{l}, \underline{m})$, and

$$
\psi(\kappa, \theta)=\frac{1}{2 \sigma_{1}(\kappa) \sigma_{2}(\kappa)} e^{-\beta}
$$

$$
\begin{gathered}
\beta=\frac{x_{\varsigma} \cos \left(\theta+\frac{\pi}{2}\right)-y_{\varsigma} \sin \left(\theta+\frac{\pi}{2}\right)}{2 \sigma_{1}(\kappa)^{2}}+\frac{x_{\varsigma} \sin \left(\theta+\frac{\pi}{2}\right)+y_{\varsigma} \cos \left(\theta+\frac{\pi}{2}\right)}{2 \sigma_{2}(\kappa)^{2}} \\
\sigma_{1}(\kappa)=\sigma_{1, \text { min }}+\alpha \kappa\left(\sigma_{1, \text { max }}-\sigma_{1, \text { min }}\right) \\
\sigma_{2}(\kappa)=\sigma_{2, \text { min }}+\kappa\left(\sigma_{2, \text { max }}-\sigma_{2, \text { min }}\right)
\end{gathered}
$$

where $\sigma_{n, \max }$ and $\sigma_{n, \min }$ are the maximum and minimum values of $\sigma_{n}$ respectively, and $\alpha$ is a shrinkage factor where $\alpha \geq 1$. By fine-tuning the weighing function based on the perceptual characteristics of each pixel individually, the noise suppression performance of the non-local means denoising technique can be improved without degrading the perceptually significant detail in the image.

\section{EXPERIMENTAL RESULTS}

To test the effectiveness of the proposed algorithm for improved perceptual quality over the non-local means (NLM) denoising algorithm, a test set of four images were used. White Gaussian noise was applied to each of the test images. For testing purposes, local neighborhoods of size $7 \times 7$ was used. To evaluate the performance of the proposed algorithm in a quantitative manner, the peak-to-peak signal to noise ratio (PSNR) was computed. The isotropic non-local means denoising algorithm was also evaluated for comparison purposes. The PSNR results are summarized in Table 1. The proposed algorithm produces higher PSNR than the isotropic non-local means denosing algorithm for all of the test cases. Example denoised images are shown in Figure 1, Figure 3, Figure 2, and Figure 4. It can be observed that the proposed algorithm produces images with noticeably better perceptual quality than the isotropic non-local means denoising algorithm. The perceptual details in the denoised images produced by the proposed algorithm are better preserved while still maintaining a high level of noise suppression.

Table 1. PSNR for Test Images

\begin{tabular}{|c|c|c|c|}
\hline \multirow{2}{*}{ Test } & \multicolumn{2}{|c|}{ PSNR (dB) } & \multirow{2}{*}{} \\
\cline { 2 - 3 } & $\begin{array}{c}\text { Isotropic NLM } \\
\text { Algorithm }\end{array}$ & $\begin{array}{c}\text { Proposed } \\
\text { Algorithm }\end{array}$ & PSNR \\
Gain \\
\hline TEST1 (23.1047 dB) & 27.3062 & 28.4433 & +1.1371 \\
TEST2 (22.8748 dB) & 26.5609 & 27.0750 & +0.5141 \\
TEST3 (16.0456 dB) & 17.3089 & 18.7122 & +1.4033 \\
TEST4 (23.1857 dB) & 26.8599 & 27.3393 & +0.4794 \\
\hline
\end{tabular}

\section{CONCLUSIONS}

This paper introduced a novel perceptually-adaptive approach to image denoising using anisotropic non-local means. By 


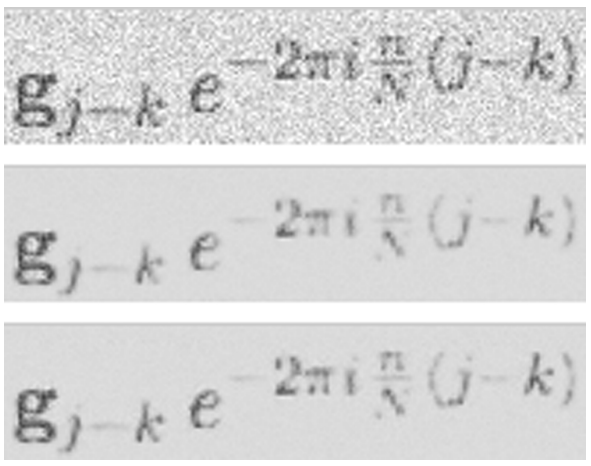

Fig. 1. TEST1: Top: Noisy image $(\sigma=0.005)$, Middle: Isotropic NLM algorithm, Bottom: Proposed algorithm
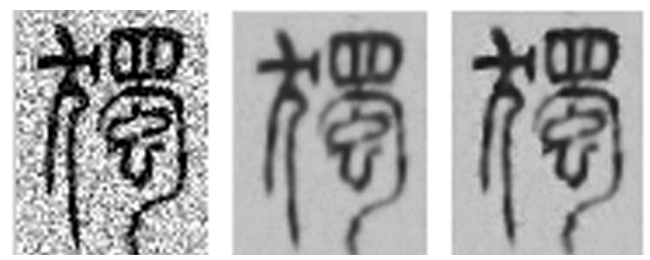

Fig. 2. TEST3: Left: Noisy image $(\sigma=0.005)$, Middle: Isotropic NLM algorithm, Right: Proposed algorithm

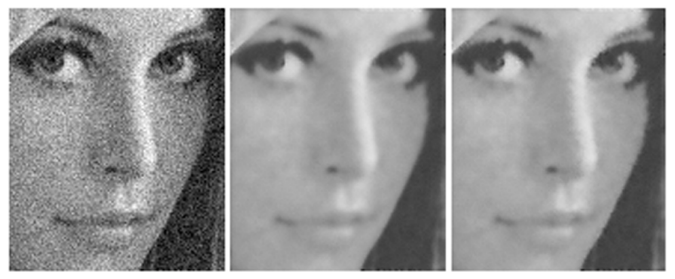

Fig. 3. TEST2: Left: Noisy image $(\sigma=0.005)$, Middle: Isotropic NLM algorithm, Right: Proposed algorithm
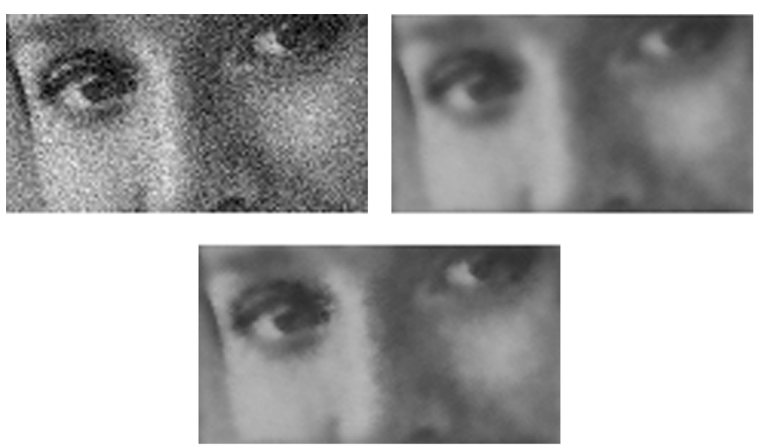

Fig. 4. TEST4: Top: Noisy image $(\sigma=0.005)$, Middle: Isotropic NLM algorithm, Bottom: Proposed algorithm adapting the size, shape, and orientation of the weighing function in an anisotropic manner based on the perceptual characteristics of the underlying image content, it was shown that noticeably improved perceptual quality can be achieved over the existing isotropic non-local means denoising algorithm. Future work includes investigating alternative objective measures for perceptual significance as well as different weighing functions.

\section{Acknowledgment}

The authors would like to thank the Natural Sciences and Engineering Research Council (NSERC) of Canada for funding this project. The authors would also like to thank the Vision and Image Processing Group at the University of Waterloo.

\section{REFERENCES}

[1] S. Greenberg and D. Kogan, "Improved structureadaptive anisotropic filter", Pattern Recognition Letters, vol. 27 , no. 1, pp. 59-65, 2006.

[2] L. Rudin and S. Osher, "Total Variation based image restoration with free local constraints", Proceedings of IEEE International Conference on Image Processing, vol. 1, pp. 31-35, 1994.

[3] C. Tomasi and R. Manduchi, "Bilateral Filtering for Gray and Color Images", Proceedings of ICCV, pp. 836-846, 1998.

[4] M. Elad, "On the origin of the bilateral filter and ways to improve it", IEEE Transactions on Image Processing, vol. 11, no. 10, 1141-1151, 2002.

[5] A. Buades, B. Coll, and J. Morel, "Nonlocal image and movie denoising," International Journal of Computer Vision, vol. (to appear), 2007.

[6] M. Mahmoudi and G. Sapiro, "Fast image and video denoising via nonlocal means of similar neighborhoods," IEEE Signal Processing Letters, vol. 12, no. 12, pp. 839842, December 2005.

[7] N. Wiener, "Extrapolation, Interpolation, and Smoothing of Stationary Time Series", New York: Wiley, 1949.

[8] J. Portilla, V. Strela, M. Wainwright, M., and E. Simoncelli, "Image denoising using scale mixtures of Gaussians in the wavelet domain", IEEE Transactions on Image Processing, vol. 12, no. 11, pp. 1338-1351, 2003.

[9] Q. Li and C. He, "Application of Wavelet Threshold to Image De-noising," In Proceedings of ICICIC, vol. 2, pp. 693-696, 2006. 